\title{
Macular thicknesses in patients with keratoconus: An optical coherence tomography study
}

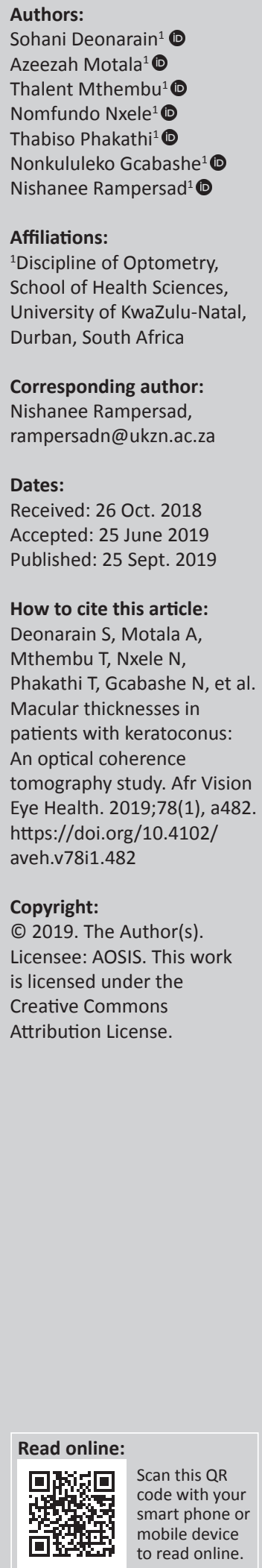

Background: Keratoconus, a corneal ectasia associated with thickness and structural changes, has been reported to co-exist with posterior segment ocular conditions. However, very few studies have reported on macular thicknesses in individuals with keratoconus.

Aim: The aim of this study was to investigate macular thicknesses in participants with keratoconus.

Setting: This study was conducted at the University of KwaZulu-Natal (UKZN).

Methods: A comparative cross-sectional research design was used. The sample consisted of 88 participants with 44 each in the control and keratoconus $(15,11$ and 18 with mild, moderate and severe keratoconus, respectively) groups. Macular thicknesses were obtained with the Fourierdomain Optovue iVue100 optical coherence tomographer using the nine Early Treatment Diabetic Retinopathy Study sectors. Data were analysed using descriptive and inferential statistics.

Results: Overall, the mean macular thicknesses were comparable among the control and three keratoconus groups $(p \geq 0.199)$. The mean central foveal thickness was greater in the severe keratoconus group $(259 \mu \mathrm{m})$ than the other three study groups that showed similar measurements $(247 \mu \mathrm{m}-248 \mu \mathrm{m})$. The central fovea was thinnest followed by the perifovea and parafovea in all four study groups. The mean thickness in the nasal and temporal quadrants of the parafovea and perifovea was thickest and thinnest, respectively, in all four study groups.

Conclusion: Macular thicknesses via optical coherence tomography in individuals with keratoconus and controls are similar with thickness differences that are clinically insignificant. Consequently, macular thicknesses should be included in the preoperative assessment of individuals with keratoconus awaiting corneal transplantation to assess the integrity of the retina prior to surgery.

Keywords: macular thickness; keratoconus; optical coherence tomography; corneal ectasia; parafoveal; peripheral retinal thickness.

\section{Introduction}

Keratoconus is a bilateral non-inflammatory progressive disease characterised by corneal thickness and structural changes. ${ }^{1,2}$ This corneal ectasia typically begins during puberty and has a prevalence of 1 in 2000 individuals with no gender and/or race predilection. ${ }^{1}$ Even though the exact pathophysiology of keratoconus is unknown, the corneal thinning characteristically associated with keratoconus is thought to be because of histopathological changes, including a partial or complete loss of the anterior limiting lamina and stromal lamellae, elongated spindleshaped superficial epithelial cells and flattened basal cells., ${ }^{3,4}$ Usually, the corneal thinning in keratoconus is localised within an ectatic region that includes the central and paracentral cornea. ${ }^{5,6,7}$ However, studies using light and electron microscopy as well as corneal mapping techniques have also shown peripheral corneal changes, albeit to a lesser degree than central corneal changes, in individuals with keratoconus.,

The alteration and thinning of the corneal stroma and epithelium in keratoconus leads to corneal protrusion and transfiguration to a conical shape. ${ }^{1}$ Consequently, astigmatism (regular and/or irregular) is a common characteristic of the refractive error in individuals with keratoconus..$^{2,5,8}$ In addition to astigmatism, other factors such as progressive myopia, higher order aberrations and media opacities contribute to visual impairment in individuals with keratoconus. ${ }^{6,8,9,10}$ The refraction characteristics and structural changes to the optical surfaces in keratoconus are likely to influence the clinical eye examination of affected individuals particularly for tests aimed at assessing the health of internal structures such as the retina. ${ }^{11}$ Various techniques can be used to examine the retina, including ophthalmoscopy (direct and indirect), slit lamp biomicroscopy with 
a condensing lens (90 D [dioptre] lens) or a contact lens (Goldmann three-mirror lens), fundus photography, fundus angiography, ultrasonography, computed tomography and optical coherence tomography (OCT) ${ }^{6,12}$

Optical coherence tomography, which is a biological imaging technology, was developed by researchers at the Massachusetts Institute of Technology in $1991 .^{13}$ Since its development, OCT has been documented as a useful technology to image biological tissues and structures in the urinary, ${ }_{1}^{14}$ respiratory, ${ }^{15}$ gastrointestinal ${ }^{16}$ and circulatory ${ }^{17}$ systems, and in recent years it has become an established method for ocular examination. ${ }^{18,19}$ This non-invasive technology allows for non-contact imaging capable of producing high-resolution cross-sectional images of structures in the anterior and posterior ocular segments. ${ }^{20,21}$ As a result, OCT is being used to image ocular structures in cases of corneal diseases including ectasia disorders and dystrophies, ${ }^{22}$ macular diseases including oedema, holes and age-related degenerations, ${ }^{23}$ and glaucoma. ${ }^{24}$ In addition to qualitative ocular imaging, OCT devices can also provide quantitative measurements of ocular structures and have shown good reliability for repeated measurements of anterior and posterior ocular segment variables. ${ }^{25,26}$

It is well known that keratoconus is associated with changes in the anterior ocular segment and studies have reported on the variable measurements therein for individuals with keratoconus. ${ }^{27,28}$ Although the optical quality of the cornea is altered in keratoconus, devices based on the principles of OCT allow for comprehensive evaluation of the corneal thickness profile in affected individuals. ${ }^{7,27}$ It has been theorised that the ocular structures in the two segments of the eye are structurally related owing to the continuity of some of the ocular structures from the anterior segment into the posterior segment. ${ }^{29}$ As keratoconus has been reported with other ocular conditions, including retinitis pigmentosa, ${ }^{30}$ Leber's congenital amaurosis, ${ }^{31}$ choroidal neovascularisation, ${ }^{32}$ central serous chorioretinopathy ${ }^{33}$ and Goldmann-Favre disease, ${ }^{34}$ investigating the posterior ocular segment in individuals with keratoconus is important particularly when corneal transplantation (penetrating keratoplasty and/or deep anterior lamellar keratoplasty) is considered as a form of management. ${ }^{32,35}$ Therefore, the aim of this study was to investigate macular thicknesses in patients with keratoconus.

\section{Methodology}

The study used a comparative cross-sectional research design and was conducted at the University of KwaZulu-Natal (UKZN) optometry clinic. Participants with keratoconus were recruited using convenience sampling and consisted of individuals who attended the UKZN optometry contact lens clinic. The control participants, who were age-, gender- and race-matched to the participants with keratoconus, were recruited using purposive sampling from individuals who attended the UKZN optometry general clinic. The keratoconus and control participants were aged between 18 and 35 years to minimise possible influences of increasing age and/or systemic diseases on the measurements of macular thickness. ${ }^{36,37}$

All participants underwent screening to assess eligibility for the study according to the study criteria. The screening procedures included case history, logarithm of the minimum angle of resolution (LogMAR) distance visual acuity, distance refraction, ophthalmoscopy, slit lamp biomicroscopy and tonometry using the iCare rebound tonometer. Participants presenting with ocular pathology other than keratoconus, systemic conditions, who are currently on medication, have a history of ocular surgery and/or injury, have intraocular pressure higher than $21 \mathrm{mmHg}$ and central corneal scarring were excluded. Participants who passed the screening procedures and satisfied the inclusion criteria proceeded to data collection, which included corneal power and macular thickness measurements. Corneal power, along the two principal meridians ( $\mathrm{K} 1$ and $\mathrm{K} 2$ ), was obtained using the Oculus Keratograph that is a reliable instrument. ${ }^{38,39}$ Macular thicknesses were obtained using OCT that has shown good repeatability and reproducibility for repeated measurements of retinal thickness in normal individuals and individuals with ocular pathologies. ${ }^{40,41}$

Participants were diagnosed with keratoconus based on slit lamp and corneal topography examinations. Clinical signs of keratoconus on the slit lamp examination included the presence of Munson's sign, Rizzuti's sign, Fleischer's ring, Vogt striae, prominent corneal nerve fibres, corneal thinning and/or scarring. The severity of keratoconus was graded using the Collaborative Longitudinal Evaluation of Keratoconus (CLEK) study classification system. ${ }^{42}$ In the CLEK classification system, which is based on the steepest corneal power measurement, the grade of keratoconus may be classified as mild (less than $45 \mathrm{D}$ ), moderate (between $45 \mathrm{D}$ and $52 \mathrm{D}$ ) or severe (greater than $52 \mathrm{D}){ }^{42}$

The OCT examination was performed using the Optovue iVue100 optical coherence tomographer. The technical specifications of this OCT device include a scanning speed of 26000 axial scans per second, a frame rate of $256-4096$ axial scans per frame and a wavelength of $830 \mathrm{~nm}-850 \mathrm{~nm} .^{43}$ Moreover, this Fourier-domain OCT device has axial and transverse resolutions of $5 \mu \mathrm{m}$ and $15 \mu \mathrm{m}$, respectively. ${ }^{43}$ Retinal thickness at the macular was measured using the retinal map scan protocol that consists of a raster pattern of horizontal line scans of $6 \mathrm{~mm}$ in length. ${ }^{43}$ This raster pattern comprises 13 line scans of 512 axial scans each in addition to 7 line scans of 1024 axial scans each within a central $1.5 \mathrm{~mm}$ vertical zone. ${ }^{43}$ For the retinal thickness scans, the internal fixation target was used to minimise off-axis fixation, while the participant's eye and retinal tomogram showing the characteristic foveal depression were monitored on the laptop screen. Retinal thickness is automatically measured by the pre-programmed algorithm in the iVue100 OCT device as the distance in microns between the inner retinal (inner limiting membrane) and outer retinal (retinal pigment epithelium) boundaries. ${ }^{43}$ Repeat retinal scans were taken 
when the scan was labelled as 'poor' on the laptop screen and/or had a scan quality index of less than 40 as per the manufacturer's recommendations. ${ }^{43}$

The retinal map scan protocol displays the average macular thicknesses in a $6 \mathrm{~mm} \times 6 \mathrm{~mm}$ macular map (Figure 1). This macular map consists of nine sectors within three regions, including the centre $(0 \mathrm{~mm}-1 \mathrm{~mm})$, parafovea $(1 \mathrm{~mm}-3 \mathrm{~mm})$ and perifovea $(3 \mathrm{~mm}-5 \mathrm{~mm})$ in accordance with the Early Treatment Diabetic Retinopathy Study (ETDRS). The average thickness in the central $1 \mathrm{~mm}$ ring was recorded as the central foveal thickness. The parafoveal and perifoveal regions comprise four quadrants (superior, inferior, nasal and temporal) each. Accordingly, the mean macular thickness in the nine ETDRS sectors are displayed using a false-colour macular map (Figure 1). In this study, the average parafoveal macular thickness and the average perifoveal macular thickness, corresponding to the inner and outer macular thicknesses, respectively, were computed as the average of the four quadrants therein.

To ensure standardisation, the setting and equipment used remained the same throughout the data collection period. Moreover, the corneal power and measurements of macular thickness were performed by one researcher each. Three measurements each for corneal power and macular thickness per participant were taken and averaged. Refractive error, measured with autorefraction and subsequently refined with subjective refraction, was converted to a spherical equivalent as half the negative cylinder power added to the sphere power. ${ }^{44}$ The central corneal thickness (CCT) measurement was obtained using the iVue100 OCT device as studies using this technology have shown high levels of repeatability for corneal thickness measurements in individuals with normal corneas and those with keratoconus. ${ }^{45,46}$ The distance visual acuity and slit lamp examinations were performed by one researcher each using a high contrast LogMAR chart and Nidek CSO biomicroscope, respectively. Intraocular pressure measurements with the iCare rebound tonometer are similar to that of the Goldmann applanation tonometer in eyes with corneal anomalies, including keratoconus. ${ }^{47}$

Data were captured and analysed using the Statistical Package for Social Sciences (SPSS, version 25). Descriptive statistics included means and standard deviations. The oneway analysis of variance (ANOVA) test was used to compare ocular variable measurements among the four study groups which included the three keratoconus groups (mild, moderate and severe) and one control group. The macular thicknesses are presented in the nine ETDRS sectors as means \pm standard

Retina 3D

Good 30

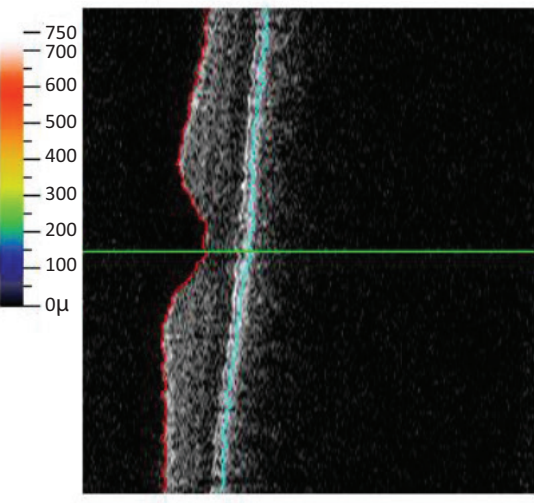

Thikcness

(2) Full

Inner

Outer

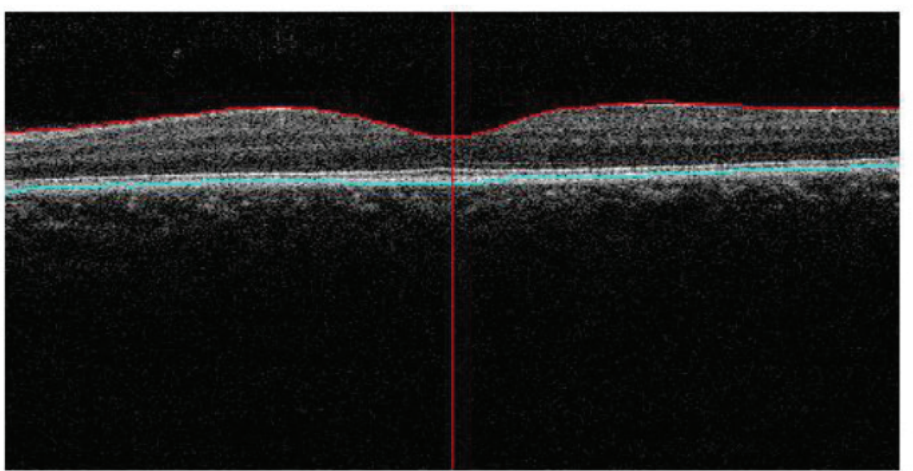

3D Display

目 
deviations. The study adopted a 95\% significance level wherein a $p$-value of 0.05 or less was considered statistically significant.

\section{Ethical considerations}

The study, with reference number BE244/17, was approved by the Biomedical Research and Ethics Committee of the University of KwaZulu-Natal (UKZN). Written informed consent was obtained from all participants and all ethical guidelines, according to the tenets of the Declaration of Helsinki, were adhered to during the study.

\section{Results}

This sample included 88 participants, 44 each in the control and keratoconus groups. In the keratoconus group, there were 15, 11 and 18 participants classified with mild, moderate and severe keratoconus, respectively. Forty-five right eyes ( 22 control, 8 mild, 6 moderate and 9 severe) and 43 left eyes (22 control, 7 mild, 5 moderate and 9 severe) were analysed. The sample included slightly more black $(n=52)$ than Indian $(n=36)$ participants. Approximately two-thirds of the participants were male $(n=56)$.

Table 1 shows the demographic and ocular characteristics of the four groups. There were no significant differences in mean age $(p=0.484)$ and intraocular pressure (IOP) $(p=$ 0.435) among the four groups. With the exception of the moderate keratoconus group, all groups had more male than female participants. The mean corneal power along the two principal meridians and astigmatism were significantly different among the four groups $(p<0.001)$, with the moderate and severe keratoconus groups showing higher values than the control and mild keratoconus groups (Table 1). Not surprisingly, the mean CCT measurements were thinner in the keratoconus groups with the lowest value $(413 \mu \mathrm{m})$ noted in the severe keratoconus group. The mean spherical equivalent refractive error differed among the four study groups $(p<0.001)$ and became significantly more myopic as the grade of keratoconus increased. The mean best-corrected

TABLE 1: Means and standard deviations for demographic and ocular characteristics in the four study groups.

\begin{tabular}{lcccc}
\hline Variables & $\begin{array}{c}\text { Controls } \\
(\boldsymbol{n}=\mathbf{4 4})\end{array}$ & \multicolumn{3}{c}{ Keratoconus groups } \\
\cline { 3 - 5 } & & Mild $(\boldsymbol{n}=\mathbf{1 5})$ & Moderate $(\boldsymbol{n}=\mathbf{1 1})$ & Severe $(\boldsymbol{n}=\mathbf{1 8})$ \\
\hline Age (years) & $24.61 \pm 4.41$ & $25.53 \pm 4.24$ & $25.45 \pm 6.30$ & $23.33 \pm 3.56$ \\
$\begin{array}{l}\text { Gender } \\
\text { (female:male) }\end{array}$ & $16: 28$ & $3: 12$ & $6: 5$ & $7: 11$ \\
$\begin{array}{l}\text { Corneal power: } \\
\text { K1 (D) }\end{array}$ & $42.44 \pm 1.67$ & $41.86 \pm 2.89$ & $45.98 \pm 1.49$ & $53.14 \pm 4.15^{*}$ \\
$\begin{array}{l}\text { Corneal power: } \\
\text { K2 (D) }\end{array}$ & $43.49 \pm 1.78$ & $43.69 \pm 1.44$ & $48.26 \pm 2.97$ & $58.26 \pm 3.86^{*}$ \\
$\begin{array}{l}\text { Corneal } \\
\text { astigmatism (D) }\end{array}$ & $0.82 \pm 0.93$ & $1.26 \pm 3.88$ & $3.76 \pm 1.63$ & $3.71 \pm 3.97^{*}$ \\
$\begin{array}{l}\text { CCT ( } \mu \text { m) } \\
\text { IOP (mmHg) }\end{array}$ & $495 \pm 29$ & $452 \pm 121$ & $470 \pm 44$ & $413 \pm 74^{*}$ \\
$\begin{array}{l}\text { Spherical } \\
\text { equivalent (D) }\end{array}$ & $-0.22 \pm 1.06$ & $-2.84 \pm 2.64$ & $-4.23 \pm 3.66$ & $-9.30 \pm 5.08^{*}$ \\
$\begin{array}{l}\text { Visual acuity } \\
\text { (LogMAR) }\end{array}$ & $-0.08 \pm 0.09$ & $0.29 \pm 0.43$ & $0.15 \pm 0.36$ & $0.31 \pm 0.21^{*}$ \\
\hline
\end{tabular}

D, dioptre; CCT, central corneal thickness; IOP, intraocular pressure.

$*, p \leq 0.05$, one-way analysis of variance test.
LogMAR visual acuity was significantly better in the control group than in the three keratoconus groups $(p<0.001)$.

Table 2 summarises the mean macular thicknesses in the four groups for the nine ETDRS sectors. Overall, there were no significant differences in the mean macular thicknesses among the four groups for the central foveal thickness ( $p=0.199)$ and each quadrant of the parafoveal $(p \geq 0.200)$ and perifoveal $(p \geq 0.211)$ regions. The mean central foveal thickness was $\sim 12 \mu \mathrm{m}$ higher in severe keratoconus than the other groups that showed similar measurements $(247 \mu \mathrm{m}-248 \mu \mathrm{m})$. The average parafoveal and perifoveal macular thicknesses were $310 \pm 16 \mu \mathrm{m}$ and $286 \pm 12 \mu \mathrm{m}, 309$ $\pm 11 \mu \mathrm{m}$ and $282 \pm 12 \mu \mathrm{m}, 304 \pm 15 \mu \mathrm{m}$ and $280 \pm 11 \mu \mathrm{m}$ as well as $313 \pm 19 \mu \mathrm{m}$ and $282 \pm 15 \mu \mathrm{m}$ in the control, mild keratoconus, moderate keratoconus as well as severe keratoconus groups, respectively. This implies that the central fovea was thinnest followed by the perifoveal and parafoveal regions in all four groups. In each quadrant of the parafoveal region, the severe and moderate keratoconus groups showed the highest and lowest mean macular thickness measurements, respectively (Table 2). Within the perifoveal region, the mild and moderate keratoconus groups showed identical macular thicknesses for all quadrants except the temporal. In all four groups, the nasal and temporal quadrants of both the parafoveal and perifoveal regions were thickest and thinnest, respectively. For the retinal scans, the mean scan quality indices were significantly better in the control group (74) than in the mild (66), moderate (62) and severe $(46)$ keratoconus groups $(p<0.001)$.

\section{Discussion}

Even though studies ${ }^{27,28}$ have reported on the measurement of ocular segment variables in individuals with keratoconus and controls, the focus has been mainly on anterior segment variables. In spite of this, the literature contains case reports of clinical instances where keratoconus has been found to coexist with some posterior segment ocular conditions. ${ }^{32,34}$ As corneal transplantation is an effective treatment option particularly for advanced keratoconus, ${ }^{5}$ a comprehensive evaluation of the posterior ocular segment is important in the clinical examination and preoperative assessment of affected individuals. In this study, macular thicknesses obtained using a Fourier-domain OCT device were compared between individuals with keratoconus and matched (age, gender

TABLE 2: Means and standard deviations for macular thickness $(\mu \mathrm{m})$ in the nine Early Treatment Diabetic Retinopathy Study sectors in the four study groups.

\begin{tabular}{lcccc}
\hline Macular region & $\begin{array}{c}\text { Control eyes } \\
(\boldsymbol{n}=\mathbf{4 4 )}\end{array}$ & \multicolumn{3}{c}{ Keratoconus groups } \\
\cline { 3 - 5 } & & $\begin{array}{c}\text { Mild } \\
(\boldsymbol{n}=\mathbf{1 5})\end{array}$ & $\begin{array}{c}\text { Moderate } \\
(\boldsymbol{n}=\mathbf{1 1})\end{array}$ & $\begin{array}{c}\text { Severe } \\
(\boldsymbol{n}=\mathbf{1 8})\end{array}$ \\
\hline Central fovea & $247 \pm 19$ & $248 \pm 7$ & $247 \pm 15$ & $259 \pm 29$ \\
Parafoveal superior & $312 \pm 16$ & $309 \pm 11$ & $303 \pm 15$ & $316 \pm 21$ \\
Parafoveal inferior & $309 \pm 15$ & $307 \pm 12$ & $304 \pm 15$ & $311 \pm 17$ \\
Parafoveal nasal & $316 \pm 18$ & $316 \pm 12$ & $314 \pm 16$ & $320 \pm 20$ \\
Parafoveal temporal & $303 \pm 16$ & $303 \pm 13$ & $293 \pm 17$ & $306 \pm 21$ \\
Perifoveal superior & $289 \pm 12$ & $282 \pm 10$ & $282 \pm 13$ & $286 \pm 19$ \\
Perifoveal inferior & $278 \pm 13$ & $273 \pm 15$ & $273 \pm 12$ & $287 \pm 20$ \\
Perifoveal nasal & $303 \pm 14$ & $299 \pm 15$ & $299 \pm 12$ & $298 \pm 16$ \\
Perifoveal temporal & $275 \pm 13$ & $272 \pm 14$ & $267 \pm 14$ & $270 \pm 16$ \\
\hline
\end{tabular}


and race) controls. Overall, the results showed that macular thicknesses were comparable among the study groups.

In this study, there was no significant difference in mean central foveal thickness measurements among the control and three keratoconus groups $(p=0.199)$. This finding is in agreement with previous studies ${ }^{11,35,48}$ that have also investigated and reported on central foveal thickness measurements between individuals with keratoconus and matched controls. Brautaset et al. ${ }^{35}$ reported an insignificant thickness difference of $\sim 4 \mu \mathrm{m}(p=0.491)$ with a Cirrus OCT device and Moschos et al. ${ }^{11}$ noted an insignificant thickness difference of $\sim 7 \mu \mathrm{m}$ ( $p=0.317)$ with a Stratus OCT device. Despite using a paediatric sample of keratoconus and control participants as well as a Spectralis OCT device, Yilmaz et al. ${ }^{48}$ reported the same trend of an insignificant central foveal thickness difference of $18 \mu \mathrm{m}(p=0.89)$ in their study. In contrast, Sahebjada et al. ${ }^{49}$ reported that the central foveal thickness measurement was $10 \mu \mathrm{m}$ significantly higher in individuals with keratoconus than controls $(p<0.05)$. Although the study by Sahebjada et al. ${ }^{49}$ consisted of a larger sample size (67 and 87 keratoconus and control participants, respectively), the participants with keratoconus differed from the controls as they were significantly younger (35 vs. 44 years, $p<0.001)$ and more likely to be male ( $80 \%$ vs. $50 \%$, $p<0.001)$. Consequently, comparing central foveal thickness measurements between individuals with keratoconus and controls without considering the influence of demographic factors such as gender and age may account for this difference. This is a possible explanation for the discrepancy in results between the present study and the study by Sahebjada et al. ${ }^{49}$ as retinal thickness measurements are lower in females and have been reported to decrease with increasing age. ${ }^{36,50}$

The mean central foveal thickness measurements were almost identical in the control $(247 \mu \mathrm{m})$, mild $(248 \mu \mathrm{m})$ and moderate $(247 \mu \mathrm{m})$ keratoconus groups. Uzunel et al. ${ }^{51}$ also noted similar central foveal thickness measurements between controls $(\sim 244 \mu \mathrm{m})$ and participants with grade 1 keratoconus $(\sim 247 \mu \mathrm{m})$, although they used a different OCT device to measure retinal thickness (Cirrus) and classification system to grade their participants with keratoconus (AmslerKrumeich system). This implies that the findings in the present study and that by Uzunel et al. ${ }^{51}$ validate the claim by Sahebjada et al. ${ }^{49}$ that retinal thickness changes in the central fovea tend to occur only in advanced stages of keratoconus. Sahebjada et al. ${ }^{49}$ proposed this claim as they observed that differences in the mean central foveal thickness measurements, between the keratoconus and control groups, failed to reach statistical significance when participants with only mild stages of keratoconus were included $(p=0.20)$. Consequently, Sahebjada et al. ${ }^{49}$ concluded that the central fovea may be unaffected in the early stages of keratoconus and starts to manifest changes with the progression of this corneal ectasia to more advanced stages.

In this study, participants in the severe keratoconus group showed $12 \mu \mathrm{m}$ higher central foveal thickness measurements than the control group ( $259 \mu \mathrm{m}$ vs. $247 \mu \mathrm{m})$. Sahebjada et al. ${ }^{49}$ postulated that the increased central foveal thickness measurements in individuals with keratoconus may be owing to a process similar to the retinomotor movements of photoreceptors observed in animal studies involving formdeprivation. These studies, which involved chick eyes with monocular form-deprivation, have reported structural and thickness changes particularly in the outer retinal layers of the form-deprived eye compared with the normal eye. ${ }^{52,53}$ By means of electron microscopy, it was shown that the photoreceptor outer segments undergo structural changes and become elongated in the form-deprived eyes. ${ }^{52}$ Some studies, involving humans with unilateral amblyopia because of anisometropia, have reported higher central macular thickness measurements in the amblyopic eye than the normal eye. ${ }^{54,55}$ Consequently, it is interesting to speculate that the central fovea becomes thicker, because of movements of the photoreceptors, to compensate for the reduced visual function in individuals with advanced keratoconus. This hypothesis, that relates to changes in the outer retina and perhaps the photoreceptor layer being accountable for the observed thickness differences, between participants with severe keratoconus and controls, is reasonable as studies have shown similar mean retinal nerve fibre layer thickness measurements in the normal and amblyopic eyes of humans with unilateral anisometropia. ${ }^{54,55}$ An alternative explanation for the increased central foveal thickness in participants with severe keratoconus may be related to a compensatory process within the eye to prevent structural disorganisation owing to the marked thinning taking place at the central cornea. ${ }^{49}$ As such, it is recommended that longitudinal studies focused on the thickness of individual layers of the retina and cornea in individuals with keratoconus should be undertaken to further investigate these speculations.

Similar to the trend for the central fovea, no significant differences in mean thickness for each quadrant in the parafovea and perifovea were observed between the control and keratoconus groups in this study $(p \geq 0.200)$. Overall, the thickness differences for the quadrants of the parafovea and perifovea ranged between $5 \mu \mathrm{m}$ and $10 \mu \mathrm{m}$ for the control and keratoconus participants. In their study, Brautaset et al..$^{35}$ reported insignificant thickness differences (range from $1.8 \mu \mathrm{m}$ to $5.2 \mu \mathrm{m}$ with $p \geq 0.122$ ) for each quadrant in the parafovea and perifovea between individuals with keratoconus and controls. Contrary to these results, Sahebjada et al. ${ }^{49}$ reported that participants with keratoconus had $10 \mu \mathrm{m}$ and $9 \mu \mathrm{m}$ significantly higher thickness measurements than controls in the parafovea and perifovea, respectively $(p<0.005)$. This implies that, irrespective of the statistical significance findings, the difference in retinal thickness for the parafovea and perifovea noted between the control and keratoconus groups in this study is in agreement with that reported in previous studies..$^{35,49}$

In addition to the comparable mean macular thicknesses among the four study groups, the characteristics of these 
measurements were also similar. For example, the mean thickness measurements were lowest for the central fovea followed by the perifovea and parafovea for all four study groups. The same trend of varying retinal thickness measurements in the macular, obtained using OCT devices, has been reported in other studies. ${ }^{36,45,56}$ Moreover, the nasal and temporal quadrants of both the parafovea and perifovea were thickest and thinnest, respectively, in all four study groups and this is in agreement with the findings of other studies. ${ }^{36,45,56}$ It has been suggested that the anatomical distribution of the fibres in the papillo-macular region accounts for this quadrant specific trend of retinal thickness measurements. ${ }^{56}$

Comparison of the mean central foveal thickness measurements found in the present study to values reported in the literature revealed interesting results. In the present study, the mean central foveal thickness measurements in participants with keratoconus ranged from $247 \mu \mathrm{m}$ to $259 \mu \mathrm{m}$. These central foveal thickness measurements are similar to the values $(243 \mu \mathrm{m}-258 \mu \mathrm{m})$ reported in other studies involving participants with keratoconus. ${ }^{10,35,48}$ In contrast, Moschos et al. ${ }^{11}$ and Sahebjada et al., ${ }^{49}$ who used a Stratus OCT device, reported lower mean central foveal thickness measurements of $\sim 158 \mu \mathrm{m}$ and $\sim 221 \mu \mathrm{m}$, respectively. This difference may be accounted for by different OCT devices using different retinal segmentation algorithms to detect the outer retinal boundary when measuring retinal thickness. This is a likely explanation as the outer retinal boundary for the iVue OCT device used in the present study is the retinal pigment epithelium layer, while the Stratus OCT device uses the junction of the inner and outer segments of the photoreceptors. ${ }^{56}$ The mean central foveal thickness in the control group was $247 \mu \mathrm{m}$ that is similar to the measurements reported in a previous study ${ }^{57}$ involving South African young adults $(238 \mu \mathrm{m}-243 \mu \mathrm{m})$ and other studies ${ }^{58,59}$ involving African-American and black adults $(245 \mu \mathrm{m}-257 \mu \mathrm{m})$.

As expected, there were significant differences in the corneal variables between the keratoconus and control groups. For example, the mean CCT thickness measurements in the keratoconus groups were thinner than that of the controls that have also been the trend in other studies. ${ }^{7,48,51}$ Even the mean corneal astigmatism, power along the two principal meridians and spherical equivalent refractive errors differed significantly among the four study groups. Overall, the three keratoconus groups had steeper corneas with higher amounts of astigmatism and myopic spherical equivalent refractive errors that corroborate the findings of other studies. ${ }^{48,49,51}$

Individuals with keratoconus present with alterations to the corneal surface where astigmatism is a common characteristic in affected individuals. ${ }^{2,5}$ In this study, the mean corneal astigmatism values were higher in the keratoconus groups than the controls. The effect of astigmatism on macular thicknesses has been evaluated in cross-sectional studies in which macular thickness was compared before and after contact lens correction as well as collagen cross-linking and with induced astigmatism. For example, Uzunel et al. ${ }^{10}$ measured retinal thickness before and after rigid gas permeable contact lenses were worn by individuals with keratoconus and found that correction of the corneal astigmatism, with rigid gas permeable contact lenses, does not have a clinically significant effect on the macular thicknesses. In another study, in which astigmatism was induced using contact lenses, it was shown that macular thicknesses were not significantly affected by induced astigmatism. ${ }^{60}$ Moreover, Romano et al. ${ }^{61}$ reported that individuals with keratoconus, who had undergone collagen cross-linking, showed no statistically significant changes in retinal morphology and the central macular thicknesses at baseline $(189 \mu \mathrm{m})$ and the 6-month follow-up $(184 \mu \mathrm{m})$ were similar. This implies that even though the mean corneal astigmatism measurements differed significantly among the four study groups, it may not have had a significant effect on the mean macular thicknesses therein.

The mean best-corrected LogMAR visual acuity was significantly better in the controls than the participants with keratoconus as has been reported previously. ${ }^{11,51}$ Brautaset et al. ${ }^{35}$ also noted better LogMAR visual acuity in controls than participants with keratoconus (0.02 vs. $0.35)$, but failed to report if this difference was statistically significant. The presence of altered media and the characteristics of the refractive error in individuals with keratoconus are likely to account for this visual acuity difference. Moschos et al., ${ }^{11}$ who compared retinal functioning by means of multifocal-electroretinogram recording in individuals with keratoconus and matched controls, observed that the electrical activity in the fovea and parafovea is significantly reduced and associated with lower best-corrected visual acuity in individuals with keratoconus. Consequently, the altered functioning despite no significant thickness changes in the macular may further account for the difference in visual acuity between participants with keratoconus and controls in this study.

In the present study, the sample composed of more males than females with keratoconus that is in agreement with previous studies. ${ }^{911,28,48,49}$ In contrast, studies ${ }^{10,51}$ involving individuals with keratoconus attending the Izmir Education and Research Hospital in Turkey reported a slightly higher percentage of females $(60 \%-65 \%)$ than males $(35 \%-40 \%)$ in their samples. This difference may be explained by a cohort effect as these two studies involved individuals with keratoconus being followed at the same institution. Although keratoconus is known to affect both males and females, ${ }^{1}$ gender-related differences with regard to vision, ocular symptoms and patient history have been reported for participants in the CLEK study. ${ }^{62}$

The scan quality index does not have a clinically significant effect on the retinal thickness measurements. ${ }^{63}$ In this study, the mean scan quality indices were significantly higher in controls than the three keratoconus groups. These results are in agreement with Brautaset et al. ${ }^{35}$ and Uzunel et al., ${ }^{51}$ who also noted significantly lower mean scan quality indices for participants with keratoconus than controls. 
Despite the lower mean scan quality indices in the three keratoconus groups (range 46 - 66), these values were still higher than the cut-off value recommended by the manufacturer (40) and it was possible to interpret all the retinal cross-sectional images as well as record the retinal thickness measurements in the present study. It is likely that the refractive and media changes in individuals with keratoconus are accountable for the lower scan quality indices observed in affected individuals. ${ }^{51}$

The strengths of this study included the use of standardised examination protocols and corneal tomography as well as topography instruments with good repeatability. Moreover, the participants with keratoconus and controls were matched for demographic characteristics (age, gender and race) to minimise the influence of the latter on the macular thickness measurements. Although the study consisted of a small sample of participants with keratoconus $(n=44)$, the evaluation and comparison of macular thicknesses among the three grades of keratoconus was considered a strength of this study. Therefore, it is recommended that future studies use larger sample sizes and a longitudinal study design to investigate macular thicknesses in individuals with differing grades of keratoconus.

\section{Conclusion}

The study concludes that the macular thickness differences between individuals with keratoconus and controls are clinically insignificant. Therefore, abnormally reduced macular thicknesses in patients with keratoconus suggest that other reasons or underlying pathologies may be present. Consequently, macular thicknesses with OCT devices should be included in the preoperative assessment of individuals with keratoconus awaiting corneal transplantation to assess the integrity of the retina prior to surgery. If any abnormal measurements are found, one needs to revaluate the need for surgery and consider that the visual outcomes after surgery may be suboptimal owing to the presence of retinal anomalies that may be affecting vision in addition to keratoconus.

\section{Acknowledgements}

The authors acknowledge Dr Sibanda from the University of KwaZulu-Natal for assistance with the statistical analysis of this study.

\section{Competing interests}

The authors declare that they have no financial or personal relationships that may have inappropriately influenced them in writing this article.

\section{Authors' contributions}

S.D. was the study leader. N.G. and N.R. provided feedback on the study design. A.M., T.M., N.N., T.P. and S.D. performed the data collection. N.R. and N.G. wrote the article.

\section{Funding information}

This research received no specific grant from any funding agency in the public, commercial or not-for-profit sectors.

\section{Data availability statement}

Data sharing is not applicable to this article.

\section{Disclaimer}

The views and opinions expressed in this article are those of the authors and do not necessarily reflect the official policy or position of any affiliated agency of the authors.

\section{References}

1. Rabinowitz YS. Keratoconus. Surv Ophthalmol. 1998;42(4):297-319. https://doi org/10.1016/S0039-6257(97)00119-7

2. Romero-Jiménez $M$, Santodomingo-Rubido J, Wolffsohn JS. Keratoconus: A review. Contact Lens Anterior Eye. 2010;33(4):157-166. https://doi.org/ 10.1016/j.clae.2010.04.006

3. Mathew JH, Goosey JD, Bergmanson JPG. Quantified histopathology of the keratoconic cornea. Optom Vis Sci. 2011;88(8):988-997. https://doi.org/10.1097/ OPX.0b013e31821ffbd4

4. Hollingsworth JG, Bonshek RE, Efron N. Correlation of the appearance of the keratoconic cornea in vivo by confocal microscopy and in vitro by light microscopy. Cornea. 2005;24(4):397-405. https://doi.org/10.1097/01.ico.0000151548.46231.27

5. Liu $\mathrm{H}$, Chen $\mathrm{Y}$, Wang $\mathrm{P}$, et al. Efficacy and safety of deep anterior lamellar keratoplasty vs. enetrating eratoplasty for eratoconus: A meta-analysis. PLoS One. 2015;10(1):e0113332. https://doi.org/10.1371/journal.pone.0113332

6. Kanski JJ. Clinical ophthalmology: A systematic approach. 6th ed. Edinburgh: Butterworth-Heinemann; 2007.

7. Brautaset RL, Nilsson M, Miller WL, Leach NE, Tukler JH, Bergmanson JPG. Centra and peripheral corneal thinning in keratoconus. Cornea. 2013;32(3):257-261. https://doi.org/10.1097/ICO.0b013e31825240d7

8. Suzuki M, Amano S, Honda N, Usui T, Yamagami S, Oshika T. Longitudinal changes in corneal irregular astigmatism and visual acuity in eyes with keratoconus. Jpn Ophthalmol. 2007;51(4):265-269. https://doi.org/10.1007/s10384-007-0453-2

9. Lim L, Wei RH, Chan WK, Tan DTH. Evaluation of higher order ocular aberrations in patients with keratoconus. J Refract Surg. 2007;23(8):825-828. https://doi. org/10.3928/1081-597X-20071001-13

10. Uzunel UD, Kusbeci T, Yuce B, Yüksel B. Effects of rigid contact lenses on optical coherence tomographic parameters in eyes with keratoconus. Clin Exp Optom. 2015;98(4):319-322. https://doi.org/10.1111/cxo.12287

11. Moschos MM, Chatziralli IP, Koutsandrea C, Siasou G, Droutsas D. Assessment of the macula in keratoconus: An optical coherence tomography and multifocal electroretinography study. Ophthalmologica. 2013;229(4):203-207. https://doi. org/10.1159/000350801

12. Bernardes $R$, Serranho $P$, Lobo $C$. Digital ocular fundus imaging: A review. Ophthalmologica. 2011;226(4):161-181. https://doi.org/10.1159/000329597

13. Huang D, Swanson EA, Lin CP, et al. Optical coherence tomography. Science. 1991;254(5035):1178-1181. https://doi.org/10.1126/science.1957169

14. Gora MJ, Suter MJ, Tearney GJ, Li X. Endoscopic optical coherence tomography: Technologies and clinical applications [Invited]. Biomed Opt Express. 2017;8(5):2405 https://doi.org/10.1364/BOE.8.002405

15. McLaughlin RA, Noble PB, Sampson DD. Optical coherence tomography in respiratory science and medicine: From airways to alveoli. Physiology. 2014;29(5):369-380. https://doi.org/10.1152/physiol.00002.2014

16. Kirtane TS, Wagh MS. Endoscopic optical coherence tomography (OCT): Advances in gastrointestinal imaging. Gastroenterol Res Pract. 2014;2014:376367. https://doi. org/10.1155/2014/376367

17. Jenkins MW, Watanabe M, Rollins AM. Longitudinal imaging of heart development with optical coherence tomography. J Sel Top Quantum Electron. 2012;18(3):1166-1175. https://doi.org/10.1109/JSTQE.2011.2166060

18. Sakata LM, DeLeon-Ortega J, Sakata V, Girkin CA. Optical coherence tomography of the retina and optic nerve -A review. Clin Exp Ophthalmol. 2009;37(1):90-99. https://doi.org/10.1111/j.1442-9071.2009.02015.x

19. Ang M, Baskaran M, Werkmeister RM, et al. Anterior segment optical coherence tomography. Prog Retin Eye Res. 2018;66:132-156. https://doi.org/10.1016/j. preteyeres.2018.04.002

20. Ramos JLB, Li Y, Huang D. Clinical and research applications of anterior segment optical coherence tomography - A review. Clin Exp Ophthalmol. 2009;37(1):81-89. https://doi.org/10.1111/j.1442-9071.2008.01823.x

21. Wojtkowski M. New directions in ophthalmic optical coherence tomography. Optom Vis Sci. 2012;89(5):524-542. https://doi.org/10.1097/OPX.0b013e31824eecb2 
22. Venkateswaran N, Galor A, Wang J, Karp CL. Optical coherence tomography for ocular surface and corneal diseases: A review. Eye Vis. 2018:5(1):13. https://doi. ocular surface and corneal diseas

23. Murthy RK, Haji S, Sambhav K, Grover S, Chalam KV. Clinical applications of spectral domain optical coherence tomography in retinal diseases. Biomed J. 2016;39(2):107-120. https://doi.org/10.1016/J.BJ.2016.04.003

24. Sathyan P, Shilpa S, Anitha A. Optical coherence tomography in glaucoma. J Cur Glaucoma Pract. 2012;6(1):1-5. https://doi.org/10.5005/jp-journals-10008-1099

25. Prakash G, Agarwal A, Jacob S, Kumar DA, Agarwal A, Banerjee R. Comparison of Fourier-domain and time-domain optical coherence tomography for assessment of corneal thickness and intersession repeatability. Am J Ophthalmol. 2009; 148(2):282.e2-290.e2. https://doi.org/10.1016/J.AJO.2009.03.012

26. Garcia-Martin E, Pinilla I, Idoipe M, Fuertes I, Pueyo V. Intra and interoperato reproducibility of retinal nerve fibre and macular thickness measurements using Cirrus Fourier-domain OCT. Acta Ophthalmol. 2011;89(1):e23-e29. https://doi. org/10.1111/j.1755-3768.2010.02045.x

27. Aurich H, Pham DT, Wirbelauer C. Biometric evaluation of keratoconic eyes with slit lamp-adapted optical coherence tomography. Cornea. 2011;30(1):56-59. https://doi.org/10.1097/ICO.0b013e3181e2ba61

28. Orucoglu F, Toker E. Comparative analysis of anterior segment parameters in normal and keratoconus eyes generated by Scheimpflug tomography. J Ophthalmol. 2015;2015:925414. https://doi.org/10.1155/2015/925414

29. Cankaya $A B$, Ozates $S$. Relationship between anterior segment and optic nerve head parameters in healthy subjects. Arq Bras Oftalmol. 2017;80(5):285-289. https://doi.org/10.5935/0004-2749.20170070

30. Moschos M, Droutsas D, Panagakis E, Tsioulias G, Tsalouki M. Keratoconus and tapetoretinal degeneration. Cornea. 1996;15(5):473-476. https://doi.org/10 1097/00003226-199609000-00006

31. Hameed $\mathrm{A}$, Khaliq $\mathrm{S}$, Ismail $\mathrm{M}$, et al. A novel locus for Leber Congenital Amaurosis (LCA4) with anterior keratoconus mapping to chromosome 17p13. Invest Ophthalmol Vis Sci. 1977;41(3):629-633.

32. Oh JY, Yu HG. Keratoconus associated with choroidal neovascularization: A case report. J Med Case Rep. 2010;4(1):58. https://doi.org/10.1186/1752-1947-4-58

33. Eandi CM, Del Priore LV, Bertelli E, Ober MD, Yannuzzi LA. Central serous chorioretinopathy in patients with keratoconus. Retina. 2008;28(1):94-96. https://doi.org/10.1097/IAE.0b013e318098629

34. Sammouh FK, Baban TA, Warrak EL. A patient with keratoconus, nanophthalmos, lipodermoids and pigmentary retinopathy. Ophthalmic Genet. 2016;37(2):228-232. https://doi.org/10.3109/13816810.2015.1028648

35. Brautaset RL, Rosén R, Cerviño A, Miller WL, Bergmanson J, Nilsson M. Comparison of macular thickness in patients with keratoconus and control subjects using the Cirrus HD-OCT. Biomed Res Int. 2015;2015:1-5. https://doi.org/10.1155/2015/ 832863

36. Song WK, Lee SC, Lee ES, Kim CY, Kim SS. Macular thickness variations with sex age and axial length in healthy subjects: A spectral domain-optical coherence to mography study. Invest Opthalmol Vis Sci. 2010;51(8):3913. https://doi org/10.1167/iovs.09-4189

37. Rao HL, Kumar AU, Babu JG, Kumar A, Senthil S, Garudadri CS. Predictors of normal optic nerve head, retinal nerve fiber layer, and macular parameters measured by spectral domain optical coherence tomography. Invest Opthalmo Vis Sci. 2011;52(2):1103. https://doi.org/10.1167/iovs.10-5997

38. Mao X, Savini G, Zhuo Z, et al. Repeatability, reproducibility, and agreement of corneal power measurements obtained with a new corneal topographer. J Cataract Refract Surg. 2013;39(10):1561-1569. https://doi.org/10.1016/J.JCRS.2013.04.029

39. Best N, Drury L, Wolffsohn JS. Clinical evaluation of the Oculus Keratograph. Contact Lens Anterior Eye. 2012;35(4):171-174. https://doi.org/10.1016/J. CLAE.2012.04.002

40. Giani A, Cigada M, Choudhry N, et al. Reproducibility of Retinal thickness measurements on normal and pathologic eyes by different optical coherence
tomography instruments. Am J Ophthalmol. 2010;150(6):815.e1-824.e1. https:// tomography instruments. Am J Opht
doi.org/10.1016/J.AJO.2010.06.025

41. Reibaldi M, Uva MG, Avitabile T, et al. Intrasession reproducibility of RNFL thickness measurements using sd-oct in eyes with keratoconus. Ophthalmic Surg Lasers Imaging. 2012;43(6):S83-S89. https://doi.org/10.3928/15428877 20121001-04

42. Zadnik K, Barr JT, Edrington TB, et al. Baseline findings in the Collaborative Longitudinal Evaluation of Keratoconus (CLEK) Study. Invest Ophthalmol Vis Sci. 1998;39(13):2537-2546.
43. Optovue. iVue100 user's manual version 1.9. Fremont, CA: Optovue Inc; 2011.

44. Stephens GL. Correction with single-vision spectacles. In: Benjamin WJ, editor Borish's clinical refraction. 2nd ed. St Louis: Butterworth-Heinemann; 2006; pp. 1026-1094.

45. Adhi M, Aziz S, Muhammad K, Adhi MI. Macular thickness by age and gender in healthy eyes using spectral domain optical coherence tomography. PLoS One. 2012;7(5):e37638. https://doi.org/10.1371/journal.pone.0037638

46. Mohamed S, Lee GKY, Rao SK, et al. Repeatability and reproducibility of pachymetric mapping with Visante anterior segment-optical coherence tomography. Invest Opthalmol Vis Sci. 2007;48(12):5499. https://doi.org/10.1167/ iovs.07-0591

47. Moreno-Montañés J, García N, Fernández-Hortelano A, García-Layana A. Rebound tonometer compared with Goldmann tonometer in normal and pathologic corneas. Cornea. 2007;26(4):427-430. https://doi.org/10.1097/ICO.0b013e318030df6e

48. Yilmaz I, Saracoglu Yilmaz B, Guleryuz NB, Perente I, Ozkaya A, Taskapili M. Assessment of the macula and choroid in pediatric keratoconus patients. Saudi $j$ Ophthalmol. 2018;32(2):126-129. https://doi.org/10.1016/J.SJOPT.2017.10.010

49. Sahebjada S, Amirul Islam FM, Wickremasinghe S, Daniell M, Baird PN. Assessment of macular parameter changes in patients with keratoconus using optical coherence tomography. J Ophthalmol. 2015;2015:245953. https://doi.org/10. coherence tomography

50. Nieves-Moreno M, Martínez-de-la-Casa JM, Morales-Fernández L, Sánchez-Jean R, Sáenz-Francés F, García-Feijoó J. Impacts of age and sex on retinal layer thicknesses measured by spectral domain optical coherence tomography with Spectralis. PLOS One. 2018;13(3):e0194169. https://doi.org/10.1371/journal. pone.0194169

51. Uzunel UD, Küsbeci T, Yüksel B. Does the stage of keratoconus affect optical coherence tomography measurements? Semin Ophthalmol. 2017;32(6):676-681. https://doi.org/10.3109/08820538.2016.1169302

52. Liang $\mathrm{H}$, Crewther D, Crewther S, Barila A. A role for photoreceptor outer segments in the induction of deprivation myopia. Vision Res. 1995;35(9):1217-1225. https://doi.org/10.1016/0042-6989(94)00241-D

53. Liang H, Crewther SG, Crewther DP, Junghans BM. Structural and elemental evidence for edema in the retina, retinal pigment epithelium, and choroid during recovery from experimentally induced myopia. Invest Opthalmol Vis Sci. 2004;45(8):2463. https://doi.org/10.1167/iovs.03-1009

54. Al-Haddad CE, Mollayess GMEL, Cherfan CG, Jaafar DF, Bashshur ZF. Retinal nerve fibre layer and macular thickness in amblyopia as measured by spectral-domain optical coherence tomography. Br J Ophthalmol. 2011;95(12):1696-1699. https:// doi.org/10.1136/bjo.2010.195081

55. Huynh SC, Samarawickrama C, Wang XY, et al. Macular and nerve fiber layer thickness in amblyopia: The Sydney Childhood Eye Study. Ophthalmology 2009;116(9):1604-1609. https://doi.org/10.1016/J.OPHTHA.2009.03.013

56. Vesela T. Mitkova-Hristova, Marieta I. Konareva-Kostyaneva. Macular thickness measurements in healthy eyes using spectral optical coherence tomography. Folia Med (Plovdiv). 2011;53(4):28-33. https://doi.org/10.2478/v10153-011-0064-z

57. Murugan C, Golodza BZ, Pillay K, et al. Retinal thickness in black and Indian myopic students at the University of KwaZulu-Natal. African Vis Eye Health. 2015;74(1):7. https://doi.org/10.4102/aveh.v74i1.300

58. Wagner-Schuman M, Dubis AM, Nordgren RN, et al. Race- and sex-related differences in retinal thickness and foveal pit morphology. Invest Opthalmol Vis Sci. 2011;52(1):625. https://doi.org/10.1167/iovs.10-5886

59. Grover S, Murthy RK, Brar VS, Chalam KV. Normative data for macular thickness by high-definition spectral-domain optical coherence tomography (Spectralis). Am Ophthalmol. 2009;148(2):266-271. https://doi.org/10.1016/J.AJO.2009.03.006

60. Hwang YH, Lee SM, Kim YY, Lee JY, Yoo C. Astigmatism and optical coherence tomography measurements. Graefe's Arch Clin Exp Ophthalmol. 2012;250(2):247254. https://doi.org/10.1007/s00417-011-1788-4

61. Romano MR, Quaranta G, Bregu M, Albe E, Vinciguerra P. No retinal morphology changes after use of riboflavin and long-wavelength ultraviolet light for treatment of keratoconus. Acta Ophthalmol. 2012;90(1):e79-e80. https://doi.org/10.1111/ j.1755-3768.2010.02067.x

62. Fink BA, Wagner $\mathrm{H}$, Steger-May K, et al. Differences in keratoconus as a function of gender. Am J Ophthalmol. 2005;140(3):459.e1-459.e12. https://doi.org/10. 1016/j.ajo.2005.03.078

63. Samarawickrama C, Pai A, Huynh SC, Burlutsky G, Wong TY, Mitchell P. Influence of OCT signal strength on macular, optic nerve head, and retinal nerve fiber layer parameters. Invest Opthalmol Vis Sci. 2010;51(9):4471. https://doi.org/10.1167/ parameters. 